\title{
Computer program for the relativistic mean field description of the ground state properties of even-even axially deformed nuclei
}

\author{
P. Ring ${ }^{1}$, Y.K. Gambhir ${ }^{1,2}$ and G.A. Lalazissis ${ }^{1,3}$ \\ ${ }^{1}$ Physik Department, Technische Universität München \\ D-85747 Garching, Germany \\ ${ }^{2}$ Physics Department, I.I.T. Powai, Bombay 400076, India \\ ${ }^{3}$ Department of Theoretical Physics, Aristotle University of Thessaloniki, \\ GR 54006 Thessaloniki, Greece.
}

February 9, 2018

\begin{abstract}
A Fortran program for the calculation of the ground state properties of axially deformed even-even nuclei in the relativistic framework is presented. In this relativistic mean field (RMF) approach a set of coupled differential equations namely the Dirac equation with potential terms for the nucleons and the Glein-Gordon type equations with sources for the meson and the electromagnetic fields are to be solved self-consistently. The well tested basis expansion method is used for this purpose. Accordingly a set of harmonic oscillator basis generated by an axially deformed potential are used in the expansion. The solution gives the nucleon spinors, the fields and level occupancies, which are used in the calculation of the ground state properties.
\end{abstract}

\section{PROGRAM SUMMARY}

Title of program: RMFD.f

Catalogue number: ??

Program available from:

Licensing provisions: none 
Computer for which the program is desinged and others on which it has been tested: any Unix work-station or mainframe or PC. The program has been tested on work stations: DEC, DEC-Alpha, on CDC6600 mainframe and on 486 IBM compatible PC's

Operating system: UNIX or VMS or MS-DOS

Programming language used: Fortran 77

No. of lines in distributed program, including test data, etc: ca. ??

Keywords: Relativistic mean field theory, binding energy, nuclear radii, deformations and densities.

Nature of physical problem

The Relativistic Mean Field (RMF) theory [1, 2] has been astonishingly succesfull [3, 4,

5, 6, 7, 8] in accurately describing the nuclear matter properties and the ground state properties of finite nuclei spread over the entire periodic table including those away from the stability line. The nucleonic and mesonic degrees of freedom are explicitly included from the very beginning in the relativistic framework. As a result the correct spin-orbit splittings emerge automatically. Initially the RMF theory was applied succesfully to the description of the properties of spherical nuclei. As most of the nuclei are deformed, the generalization of the solution of the RMF equations for this case is required, which is a non trivial task. Therefore a computer program was developed [9, 10, 3] to solve the RMF equations, suitable for the calculation of the ground state properties of the axially deformed nuclei. The present program is an improved version and also is compatible for PC's.

\section{Method of solution}

In the RMF theory one needs to solve self-consistently a set of coupled equations namely the Dirac equation with potential terms for the nucleons and the Klein-Gordon type equations with sources for the mesons and the photon. For this purpose we employ the well tested basis expansion method. The bases used here, are generated by an anisotropic (axially symmetric) harmonic oscillator potential. The upper and lower components of the nucleon spinors, the fields as well as the baryon currents and densities are expanded separately in these bases. The expansion is truncated so as to include all the configurations up to a certain finite value of the major oscillator shell quantum number. In this expansion method the solution of the Dirac equation gets reduced to a symmetric matrix diagonalization problem, while that of the Klein Gordon equation reduces to a set of inhomogenous equations. The solution provides the spinor, fields, and the nucleon currents and densities (sources of the fields), from which all the relevant ground state nuclear properties are calculated.

Restrictions on the complexity of the problem

The present version is applicable to even-even nuclei due to the imposition of time reversal inavariance and charge conservation. The program can be modified for the general case 
including that of odd mass nuclei by incorporating the additional currents arising due to time reversal breaking.

Typical running time:

From 30 minutes to several hours depending upon the computer for the general case. However, the computer time will considerably increase if one wishes to include higher number of shells.

\section{LONG WRITE-UP}

\subsection{RMF equations}

The basic Ansatz of the RMF theory is a Lagrangian density [1, 2] where nucleons are described as Dirac particles which interact via the exchange of various mesons. The Lagrangian density considered is written in the form:

$$
\begin{gathered}
\mathcal{L}=\bar{\psi}(i \not \partial-M) \psi+\frac{1}{2} \partial_{\mu} \sigma \partial^{\mu} \sigma-U(\sigma)-\frac{1}{4} \Omega_{\mu \nu} \Omega^{\mu \nu}+ \\
\frac{1}{2} m_{\omega}^{2} \omega_{\mu} \omega^{\mu}-\frac{1}{4} \vec{R}_{\mu \nu} \vec{R}^{\mu \nu}+\frac{1}{2} m_{\rho}^{2} \vec{\rho}_{\mu} \vec{\rho}^{\mu}-\frac{1}{4} F_{\mu \nu} F^{\mu \nu} \\
g_{\sigma} \bar{\psi} \sigma \psi-g_{\omega} \bar{\psi} \psi \psi-g_{\rho} \bar{\psi} \vec{\rho} \vec{\tau} \psi-e \bar{\psi} A \psi
\end{gathered}
$$

The meson fields included are the isoscalar $\sigma$ meson, the isoscalar-vector $\omega$ meson and the isovector-vector $\rho$ meson. The latter provides the necessary isospin asymmetry.

The arrows in Eq. (1) denote the isovector quantities. The Lagrangian contains also a non-linear scalar self-interaction of the $\sigma$ meson.

$$
U(\sigma)=\frac{1}{2} m_{\sigma}^{2} \sigma^{2}+\frac{1}{3} g_{2} \sigma^{3}+\frac{1}{4} g_{3} \sigma^{4}
$$

This term is important for appropriate description of surface properties [11]. $\mathrm{M}, \mathrm{m}_{\sigma}, \mathrm{m}_{\omega}$ and $\mathrm{m}_{\rho}$ are the nucleon-, the $\sigma$-, the $\omega$ - and the $\rho$-meson masses respectively, while $\mathrm{g}_{\sigma}$, $\mathrm{g}_{\omega}, \mathrm{g}_{\rho}$ and $\mathrm{e}^{2} / 4 \pi=1 / 137$ are the corresponding coupling constants for the mesons and the photon. The field tensors of the vector mesons and of the electromagnetic fields take the following form:

$$
\begin{aligned}
& \Omega^{\mu \nu}=\partial^{\mu} \omega^{\nu}-\partial^{\nu} \omega^{\mu} \\
& \vec{R}^{\mu \nu}=\partial^{\mu} \vec{\rho}^{\nu}-\partial^{\nu} \vec{\rho}^{\mu} \\
& F^{\mu \nu}=\partial^{\mu} A^{\nu}-\partial^{\nu} A^{\mu}
\end{aligned}
$$

The variational principle gives the equations of motion. The mean field approximation is introduced at this stage by treating the fields as the c-number or classical fields. This results into a set of coupled equations namely the Dirac equation with potential terms for the nucleons and the Klein-Gordon type equations with sources for the mesons and the photon. For the static case, along with the time reversal invariance and charge conservation the equations get simplified. The resulting equations, known as RMF equations have the following form. 
The Dirac equation for the nucleon:

$$
\{-i \alpha \nabla+V(\mathbf{r})+\beta[M+S(\mathbf{r})]\} \psi_{i}=\varepsilon_{i} \psi_{i},
$$

where $V(\mathbf{r})$ represents the vector potential:

$$
V(\mathbf{r})=g_{\omega} \omega_{0}(\mathbf{r})+g_{\rho} \tau_{3} \rho_{0}(\mathbf{r})+e \frac{1+\tau_{3}}{2} A_{0}(\mathbf{r}),
$$

and $S(\mathbf{r})$ is the scalar potential:

$$
S(\mathbf{r})=g_{\sigma} \sigma(\mathbf{r})
$$

the latter contributes to the effective mass as:

$$
M^{*}(\mathbf{r})=M+S(\mathbf{r}) .
$$

The Klein-Gordon equations for the meson and the electromagnetic fields with the nucleon densities as sources:

$$
\begin{aligned}
\left\{-\Delta+m_{\sigma}^{2}\right\} \sigma(\mathbf{r}) & =-g_{\sigma} \rho_{s}(\mathbf{r})-g_{2} \sigma^{2}(\mathbf{r})-g_{3} \sigma^{3}(\mathbf{r}) \\
\left\{-\Delta+m_{\omega}^{2}\right\} \omega_{0}(\mathbf{r}) & =g_{\omega} \rho_{v}(\mathbf{r}) \\
\left\{-\Delta+m_{\rho}^{2}\right\} \rho_{0}(\mathbf{r}) & =g_{\rho} \rho_{3}(\mathbf{r}) \\
-\Delta A_{0}(\mathbf{r}) & =e \rho_{c}(\mathbf{r})
\end{aligned}
$$

The corresponding densities are:

$$
\begin{aligned}
\rho_{s} & =\sum_{i=1}^{A} n_{i} \bar{\psi}_{i} \psi_{i} . \\
\rho_{v} & =\sum_{i=1}^{A} n_{i} \psi_{i}^{+} \psi_{i} . \\
\rho_{3} & =\sum_{p=1}^{Z} n_{i} \psi_{p}^{+} \psi_{p}-\sum_{n=1}^{N} n_{i} \psi_{n}^{+} \psi_{n} . \\
\rho_{c} & =\sum_{p=1}^{Z} n_{i} \psi_{p}^{+} \psi_{p} .
\end{aligned}
$$

Here the sums are taken over the particle states only. This implies that the contributions from negative-energy states are neglected (no-sea approximation), i.e. the vacuum is not polarized. The $\pi$ meson does not contribute in the present relativistic mean field (Hartree) approxiamtion because of its pseudo nature. The occupation number $n_{i}$ is introduced to account for pairing which is important for open shell nuclei. In the absence of pairing it takes the value one (zero) for the levels below (above) the Fermi surface. In the presence of pairing the partial occupancies $\left(n_{i}\right)$ are obtained in the constant gap approximation (BCS) through the well known expression:

$$
n_{i}=\frac{1}{2}\left(1-\frac{\varepsilon_{i}-\lambda}{\sqrt{\left(\varepsilon_{i}-\lambda\right)^{2}+\Delta^{2}}}\right)
$$


The $\varepsilon_{i}$ is the single-paricle energy for the state $i$ and chemical potential or Fermi energy $\lambda$ for protons (neutrons) is obtained from the requirement

$$
\begin{aligned}
\sum_{i} n_{i}= & \text { the number of protons }(\mathrm{Z}) \\
& (\text { the number of neutrons }(\mathrm{N}))
\end{aligned}
$$

The sum is taken over protons (neutrons) states. The gap parameter $\Delta$ is calculated from the observed odd-even mass differences. In the absence of experimental masses it can inferred from the extrapolation of the masses given by any of the avalaible mass formulae (e.g from ref. [12)

The above set of equations $(6,10-13)$ are to be solved self-consistently. For this purpose one starts with an initial guess of the fields (e.g. generated by axially deformed WoodsSaxon potential) to calculate the potential terms (Eqs. $(7,8)$ ) appearing in the Dirac equation (Eq. (6)). The Dirac equation is solved with these potentials terms to yield the nucleon spinors which in term are used to obtain the sources (densities). The meson and photon equations are then solved with these sources to get a new set of fields to be used for the calculation of new potential terms. The Dirac equation is then solved with the new potentials to get the spinors again to be used to obtain the new sources for the meson fields. This iterative procedure is continued till the converegence upto the desired accuracy is achieved.

\subsection{Axially symmetric case}

For the axially symmetric deformed shape the rotational symmetry is broken and therefore, the total angular momentum $j$ is no longer a good quantum number. However, the densities are still invariant with respect to a rotation around the symmetry axis, which is taken to be the z-axis. It then turns out to be useful to work with the cylindrical coordinates

$$
x=r_{\perp} \cos \varphi, \quad y=r_{\perp} \sin \varphi \text { and } z .
$$

For such nuclei the Dirac equation can be reduced to a coupled set of partial differential equations in the two variables $z$ and $r_{\perp}$. In particular, the spinor $\psi_{i}$ with the index $i$ is now characterized by the quantum numbers

$$
\Omega_{i}, \pi_{i}, t_{i}
$$

where $\Omega_{i}$ is the eigenvalue of the symmetry operator $j_{z_{i}}$ (the projection of $j_{i}$ on the z-axis), $\pi_{i}$ is the parity and $t_{i}$ is the isospin. The spinor can be written in the form:

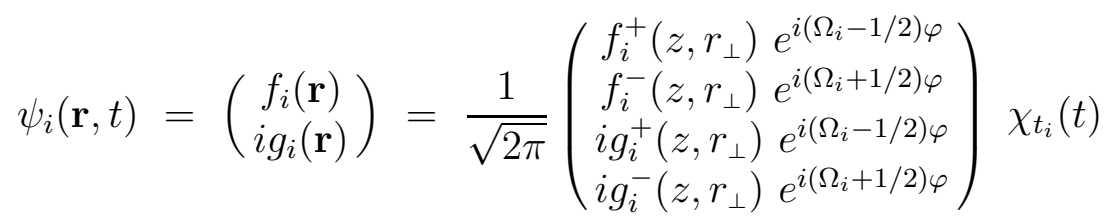


The four components $f_{i}^{ \pm}\left(r_{\perp}, z\right)$ and $g_{i}^{ \pm}\left(r_{\perp}, z\right)$ obey the Dirac equations

$$
\begin{array}{ll}
\left(M^{*}+V\right) f_{i}^{+}+\partial_{z} g_{i}^{+}+\left(\partial_{r_{\perp}}+\frac{\Omega+\frac{1}{2}}{r_{\perp}}\right) g_{i}^{-} & =\epsilon_{i} f_{i}^{+} \\
\left(M^{*}+V\right) f_{i}^{-}-\partial_{z} g_{i}^{-}+\left(\partial_{r_{\perp}}-\frac{\Omega-\frac{1}{2}}{r_{\perp}}\right) g_{i}^{+} & =\epsilon_{i} f_{i}^{-} \\
\left(M^{*}-V\right) g_{i}^{+}+\partial_{z} f_{i}^{+}+\left(\partial_{r_{\perp}}+\frac{\Omega+\frac{1}{2}}{r_{\perp}}\right) f_{i}^{-} & =-\epsilon_{i} g_{i}^{+} \\
\left(M^{*}-V\right) g_{i}^{-}-\partial_{z} f_{i}^{-}+\left(\partial_{r_{\perp}}-\frac{\Omega-\frac{1}{2}}{r_{\perp}}\right) f_{i}^{+} & =-\epsilon_{i} g_{i}^{-}
\end{array}
$$

For each solution with positive $\Omega$ :

$$
\psi_{i} \equiv\left\{f_{i}^{+}, f_{i}^{-}, g_{i}^{+}, g_{i}^{-}, \Omega_{i}\right\}
$$

we have the time reversed solution with the same energy

$$
\psi_{\bar{\iota}}=T \psi_{i} \equiv\left\{-f_{i}^{-}, f_{i}^{+}, g_{i}^{-},-g_{i}^{+},-\Omega_{i}\right\}
$$

with the time reversal operator $T=i \sigma_{y} K$ ( $K$ being the complex conjugation). For nuclei with time reversal symmetry, the contributions to the densities of the two time reversed states $i$ and $\bar{i}$ are identical. Therefore, we find the densities

$$
\rho_{s, v}=2 \sum_{i>0} n_{i}\left(\left(\left|f_{i}^{+}\right|^{2}+\left|f_{i}^{-}\right|^{2}\right) \mp\left(\left|g_{i}^{+}\right|^{2}+\left|g_{i}^{-}\right|^{2}\right)\right)
$$

and, in a similar way, $\rho_{3}$ and $\rho_{c}$. The sum $i>0$ runs only over the states with positive $\Omega_{i}$-values. These densities serve as sources for the fields $\phi=\sigma, \omega^{0} \rho^{0}$ and $A^{0}$, which are determined by the Klein-Gordon equation in cylindrical coordinates:

$$
\left(-\frac{1}{r_{\perp}} \partial_{r_{\perp}} r_{\perp} \partial_{r_{\perp}}-\partial_{z}{ }^{2}+m_{\phi}{ }^{2}\right) \phi\left(z, r_{\perp}\right)=s_{\phi}\left(z, r_{\perp}\right)
$$

The inhomogeneous parts $s_{\phi}$ are given by

$$
s_{\phi}\left(z, r_{\perp}\right)= \begin{cases}-g_{\sigma} \rho_{s}\left(z, r_{\perp}\right)-g_{2} \sigma^{2}\left(z, r_{\perp}\right)-g_{3} \sigma^{3}\left(z, r_{\perp}\right) & \text { for the } \sigma \text {-field } \\ g_{\omega} \rho_{v}\left(z, r_{\perp}\right) & \text { for the } \omega \text {-field } \\ g_{\rho} \rho_{3}\left(z, r_{\perp}\right) & \text { for the } \rho \text {-field } \\ e \rho_{p}\left(z, r_{\perp}\right) & \text { for the Coulomb field }\end{cases}
$$

\subsection{Solution of the RMF equations}

For the solution of thr RMF equations we use the basis expansion method. We follow closely the details, presentation and the notation of ref. [3]. For the axially symmetric case we expand the spinors $f_{i}^{ \pm}$and $g_{i}^{ \pm}$in Eqs. (20-23) in terms of the eigenfunctions of a deformed axially symmetric oscillator potential

$$
V_{\text {osc }}\left(z, r_{\perp}\right)=\frac{1}{2} M \omega_{z}^{2} z^{2}+\frac{1}{2} M \omega_{\perp}^{2} r_{\perp}^{2}
$$


Imposing volume conservation, the two oscillator frequencies $\hbar \omega_{\perp}$ and $\hbar \omega_{z}$ can be expressed in terms of a deformation parameter $\beta_{0}$ :

$$
\begin{aligned}
& \hbar \omega_{z}=\hbar \omega_{0} \exp \left(-\sqrt{\frac{5}{4 \pi}} \beta_{0}\right) \\
& \hbar \omega_{\perp}=\hbar \omega_{0} \exp \left(+\frac{1}{2} \sqrt{\frac{5}{4 \pi}} \beta_{0}\right)
\end{aligned}
$$

The corresponding oscillator length parameters are

$$
b_{z}=\sqrt{\frac{\hbar}{M \omega_{z}}} \text { and } \quad b_{\perp}=\sqrt{\frac{\hbar}{M \omega_{\perp}}}
$$

Because of volume conservation, we have $b_{\perp}^{2} b_{z}=b_{0}^{3}$. The basis is now determined by the two constants $\hbar \omega_{0}$ and $\beta_{0}$. The eigenfunctions of the deformed harmonic oscillator potential are characterized by the quantum numbers

$$
|\alpha>=| n_{z}, n_{r}, m_{l}, m_{s}>
$$

where $m_{l}$ and $m_{s}$ are the components of the orbital angular momentum and of the spin along the symmetry axis. The eigenvalue of $j_{z}$, which is a conserved quantity in these calculations, is

$$
\Omega=m_{l}+m_{s}
$$

The parity is given by

$$
\pi=(-)^{n_{z}+m_{l}}
$$

The eigenfunctions of the deformed harmonic oscillator can be written explicitly as

$$
\begin{array}{cl}
\Phi_{\alpha}\left(z, r_{\perp}, \varphi, s, t\right)=\quad & \phi_{n_{z}}(z) \phi_{n_{r}}^{m_{l}}\left(r_{\perp}\right) \frac{1}{\sqrt{2 \pi}} e^{i m_{l} \varphi} \chi_{m s}(s) \chi_{t_{\alpha}}(t) \\
= & \Phi_{\alpha}(\mathbf{r}, s) \chi_{t_{\alpha}}(t)
\end{array}
$$

with

$$
\begin{aligned}
\phi_{n_{z}}(z) & =\frac{N_{n_{z}}}{\sqrt{b_{z}}} H_{n_{z}}(\zeta) e^{-\zeta^{2} / 2} \\
\phi_{n_{r}}^{m_{l}}\left(r_{\perp}\right) & =\frac{N_{n_{r}}^{m_{l}}}{b_{\perp}} \sqrt{2} \eta^{m_{l} / 2} L_{n_{r}}^{m_{l}}(\eta) e^{-\eta / 2}
\end{aligned}
$$

and

$$
\zeta=z / b_{z}, \quad \eta=r_{\perp}^{2} / b_{\perp}^{2}
$$

The polynomials $H_{n}(\zeta)$ and $L_{n}^{m}(\eta)$ are Hermite polynomials and associated Laguerre polynomials as defined in ref. [13] The normalization constants are given by

$$
N_{n_{z}}=\frac{1}{\sqrt{\sqrt{\pi} 2^{n_{z}} n_{z} !}} \text { and } N_{n_{r}}^{m_{l}}=\sqrt{\frac{n_{r} !}{\left(n_{r}+m_{l}\right) !}}
$$

In order to evaluate the matrix elements, we also need the polynomials $\tilde{L}_{n_{r}}^{m_{l}}$ and $\tilde{H}_{n_{z}}$ 
defined by the derivatives

$$
\begin{aligned}
\partial_{z} \phi_{n_{z}}(z) & =\frac{N_{n_{z}}}{b_{z}^{3 / 2}} \tilde{H}_{n_{z}}(\zeta) e^{-\zeta^{2} / 2} \\
\partial_{r_{\perp}} \phi_{n_{r}}^{m_{l}}\left(r_{\perp}\right) & =\frac{N_{n_{r}}^{m_{l}}}{b_{\perp}^{2}} \sqrt{2} \eta^{\left(m_{l}-1\right) / 2} \tilde{L}_{n_{r}}^{m_{l}}(\eta) e^{-\eta / 2}
\end{aligned}
$$

which can be calculated from the recursion relations

$$
\begin{aligned}
\tilde{H}_{n_{z}}(\zeta) & =\zeta H_{n_{z}}(\zeta)-H_{n_{z}+1}(\zeta) \\
\tilde{L}_{n_{r}}^{m_{l}}(\eta) & =\left(2 n_{r}+m_{l}-\eta\right) L_{n_{r}}^{m_{l}}(\eta)-2\left(n_{r}+m_{l}\right) L_{n_{r}-1}^{m_{l}}(\eta)
\end{aligned}
$$

The solutions of the Dirac equation in the axially symmetric case have only the good quantum numbers $\Omega$ and $\pi$ and we use the expansion

$$
\begin{aligned}
& f_{i}(\mathbf{r}, s, t)=\frac{1}{\sqrt{2 \pi}}\left(\begin{array}{c}
f_{i}^{+}\left(z, r_{\perp}\right) e^{i(\Omega-1 / 2) \varphi} \\
f_{i}^{-}\left(z, r_{\perp}\right) e^{i(\Omega+1 / 2) \varphi}
\end{array}\right)=\sum_{\alpha}^{\alpha_{\max }} f_{\alpha}^{(i)} \Phi_{\alpha}(\mathbf{r}, s) \chi_{t_{i}}(t) \\
& g_{i}(\mathbf{r}, s, t)=\frac{1}{\sqrt{2 \pi}}\left(\begin{array}{c}
g_{i}^{+}\left(z, r_{\perp}\right) e^{i(\Omega-1 / 2) \varphi} \\
g_{i}^{-}\left(z, r_{\perp}\right) e^{i(\Omega+1 / 2) \varphi}
\end{array}\right)=\sum_{\tilde{\alpha}}^{\tilde{\alpha}_{\max }} g_{\tilde{\alpha}}^{(i)} \Phi_{\tilde{\alpha}}(\mathbf{r}, s) \chi_{t_{i}}(t)
\end{aligned}
$$

To avoid the appearence of the spurious states [3, 14] the quantum numbers $\alpha_{\max }$ and $\tilde{\alpha}_{\max }$ are chosen in such a way that the corresponding major quantum numbers $N=$ $n_{z}+2 n_{\rho}+m_{l}$ are not larger than $N_{F}+1$ for the expansion of the small components, and not larger than $N_{F}$ for the expansion of the large components. The Dirac equation reduces to a symmetric matrix diagonalization problem:

$$
\left(\begin{array}{cc}
\mathcal{A}_{\alpha, \alpha^{\prime}} & \mathcal{B}_{\alpha, \tilde{\alpha}^{\prime}} \\
\mathcal{B}_{\tilde{\alpha}, \alpha^{\prime}} & -\mathcal{C}_{\tilde{\alpha}, \tilde{\alpha}^{\prime}}
\end{array}\right)\left(\begin{array}{c}
f_{\alpha^{\prime}}^{(i)} \\
g_{\tilde{\alpha}^{\prime}}^{(i)}
\end{array}\right)=\epsilon_{i}\left(\begin{array}{c}
f_{\alpha}^{(i)} \\
g_{\tilde{\alpha}}^{(i)}
\end{array}\right)
$$

of the dimension $\alpha_{\max }+\tilde{\alpha}_{\max }$. The matrix elements $\mathcal{A}_{\alpha \alpha^{\prime}}, \mathcal{B}_{\alpha \alpha^{\prime}}$ and $\mathcal{C}_{\alpha \alpha^{\prime}}$ are given by:

$$
\begin{aligned}
& \left(\begin{array}{c}
\mathcal{A}_{\alpha \alpha^{\prime}} \\
\mathcal{C}_{\alpha \alpha^{\prime}}
\end{array}\right)=\delta_{m_{l} m_{l}{ }^{\prime}} \delta_{m_{s} m_{s}^{\prime}} \quad N_{n_{r}}^{m_{l}} N_{n_{z}} N_{n_{r^{\prime}}}^{m_{l^{\prime}}} N_{n_{z^{\prime}}} \int_{0}^{\infty} d \eta e^{-\eta} \eta^{m_{l}} L_{n_{r}}^{m_{l}}(\eta) L_{n_{r^{\prime}}}^{m_{l}}(\eta) \\
& \times \int_{0}^{\infty} d \zeta e^{-\zeta^{2}} H_{n_{z}}(\zeta) H_{n_{z^{\prime}}}(\zeta)\left(M^{*}\left(b_{z} \zeta, b_{\perp} \sqrt{\eta}\right) \pm V\left(b_{z} \zeta, b_{\perp} \sqrt{\eta}\right)\right) \\
& \mathcal{B}_{\alpha \alpha^{\prime}}=\delta_{m_{l} m_{l}{ }^{\prime}} \delta_{m_{s} m_{s}^{\prime}} \delta_{n_{r} n_{r^{\prime}}} \frac{(-)^{\frac{1}{2}-m_{s}}}{b_{z}}\left(\delta_{n_{z^{\prime} n_{z}+1}} \sqrt{\frac{n_{z}{ }^{\prime}}{2}}-\delta_{n_{z} n_{z^{\prime}}+1} \sqrt{\frac{n_{z}}{2}}\right) \\
& +\delta_{m_{l} m_{l}{ }^{\prime}} \delta_{n_{z} n_{z^{\prime}}} \frac{N_{n_{r}}^{m_{l}} N_{n_{r^{\prime}}}^{m_{l^{\prime}}}}{b_{\perp}}\{ \\
& \delta_{m_{s}^{\prime} m_{s}+1} \int_{0}^{\infty} d \eta e^{-\eta} \eta^{m_{l}-1 / 2} L_{n_{r}}^{m_{l}}(\eta)\left(\tilde{L}_{n_{r}^{\prime}}^{m_{l}}(\eta)-m_{l} L_{n_{r}}^{m_{l}}(\eta)\right) \\
& \left.+\quad \delta_{m_{s} m_{s}^{\prime}+1} \int_{0}^{\infty} d \eta e^{-\eta} \eta^{m_{l}-1 / 2} L_{n_{r}}^{m_{l}}(\eta)\left(\tilde{L}_{n_{r^{\prime}}}^{m_{l}}(\eta)+\left(m_{l}+1\right) L_{n_{r}}^{m_{l}}(\eta)\right)\right\}
\end{aligned}
$$


In the next step, we calculate the density matrices in the shell model space:

$$
\rho_{\alpha \alpha^{\prime}}^{s, v}=2 \sum_{i>0} n_{i}\left(f_{\alpha}^{(i)} f_{\alpha^{\prime}}^{(i)} \mp g_{\alpha}^{(i)} g_{\alpha^{\prime}}^{(i)}\right)
$$

and in coordinate space:

$$
\begin{aligned}
\rho_{s, v}\left(z, r_{\perp}\right)= & \frac{1}{b_{0}^{3}} \frac{1}{\pi} e^{-\zeta^{2}-\eta} \sum_{\alpha \alpha^{\prime}} \rho_{\alpha \alpha^{\prime}}^{s, v} \quad \delta_{m_{l} m_{l^{\prime}}} \\
& \times N_{n_{z}} N_{n_{z^{\prime}}} H_{n_{z}}(\zeta) H_{n_{z^{\prime}}}(\zeta) N_{n_{r}}^{m_{l}} N_{n_{r^{\prime}}}^{m_{l}} \eta^{m_{l}} L_{n_{r}}^{m_{l}}(\eta) L_{n_{r^{\prime}}}^{m_{l}}(\eta) .
\end{aligned}
$$

Similarly, one obtains $\rho_{3}$ and $\rho_{c}$. These are the sources for the solution of the Klein-Gordon equations. The fields of massive mesons are also solved by the expansion in a deformed oscillator basis. Here for computational and numerical convenience we use the same deformation parameter $\beta_{0}$ as in Eqs $(32,33)$, and take the oscillator length $b_{B}=b_{0} / \sqrt{2}$ :

$$
\phi\left(z, r_{\perp}\right)=\frac{1}{b_{B}^{3 / 2}} e^{-\zeta^{2} / 2-\eta / 2} \sum_{n_{z} n_{r}}^{N_{B}} \phi_{n_{z} n_{r}} N_{n_{z}} H_{n_{z}}(\zeta) \sqrt{2} L_{n_{r}}^{0}(\eta)
$$

with $\zeta=\sqrt{2} z / b_{z}$ and $\eta=2 r_{\perp}^{2} / b_{\perp}^{2}$. Inserting this ansatz into the Klein-Gordon equation, we obtain an inhomogeneous set of linear equations

$$
\sum_{n_{z^{\prime} n_{r}}}^{N_{B}} \mathcal{H}_{n_{z} n_{r} n_{z^{\prime} n_{r}}} \phi_{n_{z^{\prime} n_{r}}}=s_{n_{z} n_{r}}^{\phi}
$$

with the matrix elements

$$
\begin{aligned}
\mathcal{H}_{n_{z} n_{r} n_{z^{\prime} n_{r^{\prime}}}} & =\left(-\frac{2}{b_{z}^{2}}\left(n_{z}+1 / 2\right)-\frac{2}{b_{\perp}^{2}}\left(2 n_{r}+1\right)+m_{\phi}^{2}\right) \delta_{n_{r} n_{r}} \delta_{n_{z} n_{z^{\prime}}} \\
& +\frac{2}{b_{z}^{2}}\left(\frac{1}{2} \sqrt{\left(n_{z}+1\right) n_{z}{ }^{\prime}} \delta_{n_{z} n_{z}+1}+\frac{1}{2} \sqrt{\left(n_{z}{ }^{\prime}+1\right) n_{z}} \delta_{n_{z} n_{z^{\prime}}+1}\right) \\
& -\frac{2}{b_{\perp}^{2}}\left(n_{r}{ }^{\prime} \delta_{n_{r^{\prime} n_{r}+1}}+n_{r} \delta_{n_{r} n_{r^{\prime}+1}}\right)
\end{aligned}
$$

For the Coulomb field, due to its long range character we cannot use the oscillator basis expansion method. Therefore for this case the standard Green's function method is used as is discussed in the appendix of ref. 15

In the present case with time reversal symmetry and pairing the total binding energy is given by the sum of various individual contributions:

$$
E=E_{\text {part }}+E_{\sigma}+E_{\omega}+E_{\rho}+E_{c}+E_{\text {pair }}+E_{C M}-A M
$$

with

$$
\begin{aligned}
E_{\text {part }} & =\sum_{i} n_{i}{ }^{2} \epsilon_{i} \\
E_{\sigma L} & =-\frac{g_{\sigma}}{2} \int d^{3} r \rho_{s}(\mathbf{r}) \sigma(\mathbf{r})
\end{aligned}
$$




$$
\begin{aligned}
E_{\sigma N L} & =-\frac{1}{2} \int d^{3} r\left\{\frac{2}{3} g_{2} \sigma(\mathbf{r})^{3}+\frac{1}{2} g_{3} \sigma(\mathbf{r})^{4}\right\} \\
E_{\omega} & =-\frac{g_{\omega}}{2} \int d^{3} r \rho_{v}(\mathbf{r}) \omega^{0}(\mathbf{r}) \\
E_{\rho} & =-\frac{g_{\rho}}{2} \int d^{3} r \rho_{3}(\mathbf{r}) \rho^{00}(\mathbf{r}) \\
E_{c} & =-\frac{e^{2}}{8 \pi} \int d^{3} r \rho_{c}(\mathbf{r}) A^{0}(\mathbf{r}) \\
E_{\text {pair }} & =-\Delta \sum_{i} \sqrt{n_{i}\left(1-n_{i}\right)} \\
E_{C M} & =-\frac{3}{4} \hbar \omega_{0}=-\frac{3}{4} 41 A^{-\frac{1}{3}}
\end{aligned}
$$

In the calculation of the pairing energy we use a pairing window, i.e. the sum over $i$ in Eq. (69) is only extended up to the level where $\epsilon_{i}-\lambda \leq 2\left(41 A^{-\frac{1}{3}}\right)$. The factor 2 has been determined so as to reproduce the pairing correlation energy (Eq. (69)) for neutrons in the nucleus ${ }^{118} \mathrm{Sn}$ calculated by using the Gogny force [16].

The charge radius is calculated using the following formula:

$$
r_{c}=\sqrt{r_{p}^{2}+0.64} \quad(f m)
$$

The factor 0.64 in Eq. (71) accounts for the finite size effects of the proton.

The quadrupole $Q_{n, p}$ and hexadecupole $H_{n, p}$ moments for neutrons and protons are calculated using the expressions:

$$
Q_{n, p}=<2 r^{2} P_{2}(\cos \theta)>_{n, p}=<2 z^{2}-x^{2}-y^{2}>_{n, p}
$$

and

$$
H_{n, p}=<r^{4} Y_{40}(\theta)>_{n, p}=\sqrt{\frac{9}{64 \pi}}<8 z^{4}-24 z^{2}\left(x^{2}+y^{2}\right)+3\left(x^{2}+y^{2}\right)^{2}>_{n, p}
$$

The conventional deformation parameter $\beta$ is obtained from the calculated quadrupole moments through

$$
Q=Q_{n}+Q_{p}=\sqrt{\frac{16 \pi}{5}} \frac{3}{4 \pi} A R_{0}^{2} \beta
$$

with $R_{0}=1.2 A^{\frac{1}{3}}(\mathrm{fm})$.

\subsection{Calculation of ground state properties}

The present program has been used to calculate the ground propetries of axially deformed even-even nuclei spread over the entire periodic table encompasing also the nuclei far away from the stability line.

For carrying out explicit numerical calculations one requires the following input information: 
i) The baryon and the meson masses and the coupling constants of the meson fields to the nucleons appearing in the Lagrangian (Eq.(1))

ii) The number of oscillator shells $N_{F}$ and $N_{B}$, i.e. the cutoff major oscillator shell quantum number up to which the Dirac spinors (Fermion wavefunctions) and meson fields (describing the Bosonic degrees of freedom), and so also the densities, are expanded.

iii) The basis parameters $\hbar \omega_{0}$ and $\beta_{0}$ used for the expansion of the Dirac spinors and the fields. Following ref. [3] we fix for fermions $\hbar \omega_{0}=41 A^{-1 / 3}$ and $\beta_{0}$ can be taken to be any reasonable value (preferably close to the experimental value).

As stated before the oscillator size parameter for the meson fields (and the densities) are fixed at $1 / \sqrt{2}$ times the corresponding Fermion parameters. The deformation parameter of the oscillator basis $\beta_{0}$ has been chosen to be identical for the Dirac spinors and the meson fields. This simplifies the calculations and avoids the need for additional parameters.

For illustration we present here the results of calculations for the ground state properties of Sr nuclei over a wide range of isospin [7], namely Sr isotopes with mass numbers $A=70$ up to $A=110$. The calculations have been carried out using the Lagrangian parameter set NL-SH, which provides good results on both sides of the stability line [17]. The NL-SH set of parameters are listed in Table 1.

The number of shells taken into account in the expansion is 12 for both fermions and bosons $\left(N_{F}=N_{B}=12\right)$. It should be noted that for convergence reasons 14 shells were also considered. It was observed that, there is hardly any difference between these two sets of results. This observation also holds for nuclei in the entired periodic table. Therefore, it is sufficient to consider 12 shells in practical calculations. However, for superheavy nuclei probably one has to include higher number of shells.

For open shell nuclei, pairing has been included using the BCS formalism. In the BCS calculations we have used constant pairing gaps, which are taken from the empirical particle separation energies [18 of neighbouring nuclei.

Now we present and discuss some of the calculated results. Fig. 1 shows the binding energy per nucleon (E/A) for Sr isotopes. The empirical values taken from the 1993 Atomic Mass Evaluation Tables [18 (expt.) are also shown. The figure also includes the predictions of the recent finite-range droplet model [12] (FRDM) and of the extended ThomasFermi with Strutinsky Integral (ETF-SI) model [19] for comparison. The parabolic shapes of the binding energy per nucleon emerges nicely. The minimum in the binding energy is observed at the magic neutron number $\mathrm{N}=50$ in the RMF as well as in the mass models. The calculated RMF binding energies agree closely to the predictions of the FRDM and ETFSI models within 1-2 MeV. The RMF theory predicts binding energies which are in accord with the empirical values in almost all the cases, with deviations $\leq 0.5 \%$. It should be noted that similar type of agreement has also been obtained in other mass regions.

We have performed calculations in the RMF theory for both the prolate and oblate configurations. The deformations of nuclei have been obtained from the relativistic Hartree minimization. We show in Fig. 2 the quadrupole deformation $\beta_{2}$ for the shape corresponding to the lowest energy. The predictions of FRDM and ETF-SI are also shown for 
comparison. It is seen that the RMF theory gives a well-defined prolate shape for lighter isotopes. Further, an addition of a few neutrons below the closed neutron shell leads to an oblate shape. This shape turns into spherical ones as nuclei approach the magic neutron number $\mathrm{N}=50$. Nuclei above this magic number revert again to the prolate shape in the RMF theory. Thus, a shape transition from prolate-oblate-spherical-prolate is followed.

In addition to the lowest minimum, several isotopes exhibit a second minimum, thus implying a shape-coexistence, i.e., the prolate and the oblate shapes differ in the energy only by a few hundred keV. These nuclei have been shown by squares surrounding the black circles. The calculations predict two minima for several heavy Sr nuclei, the prolate shape results being a few hundreds keV lower in energy than the oblate one. This is displayed in Fig. 3, where the difference in the ground-state binding energy of the oblate and the prolate configurations is shown. It can be noticed that $\mathrm{Sr}$ isotopes beginning with $\mathrm{A}=92$ acquire a prolate shape predominantly. For nuclei close to $\mathrm{A}=98$, the prolate shape is lower than the oblate one only by about $300 \mathrm{keV}$. With a further increase in the neutron number the Sr isotopes take up the prolate minimum, the oblate shape being about $600-800 \mathrm{keV}$ higher. The undulation in the prolate-oblate energy differences of neutron-rich $\mathrm{Sr}$ isotopes is a noteworthy feature of the RMF prediction.

The $\beta_{2}$ values from NL-SH, FRDM and ETF-SI is shown in Table 2. The $\beta_{2}$ values for the second minimum obtained for $\mathrm{Sr}$ isotopes with the force NL-SH are shown in the parentheses. A comparison shows that the three approaches provide values close to each other. The experimental quadrupole deformations obtained from $\mathrm{BE}(2)$ values taken from [22] are shown in the last columns of the table. It may be noted that these empirical $\beta_{2}$ values do not indicate any signature as to ascertain the shape of a given nucleus. The absolute values, however, do compare well with the RMF predictions.

In Fig.4 the rms charge and neutron radii of Sr nuclei are presented. It is seen that on going from the lighter isotopes to the heavier ones the charge radii exhibit a decreasing trend upto the magic isotope, that is the lighter isotopes have higher charge radii than the heavier closed neutron- shell nucleus. The charge radii for nuclei heavier than the closed neutron-shell start increasing with addition of neutrons. The neutron radii, on the other hand, also show a kink about the neutron shell closure. However, the neutron radius for lighter isotopes in these chains is not higher than that of the closed-shell nucleus.

In Fig. 5 we show the isotope shifts $\left.r_{c}^{2}(A)-r_{c}^{2}(r e f)\right)$ for Sr nuclei calculated with respect to a reference nucleus $\left({ }^{88} \mathrm{Sr}\right)$. The empirical data obtained from atomic laser spectroscopy [20, 21] are also shown. The experimental data for Sr nuclei exhibit a kink about the magic neutron number. This kink about the closed-shell is a characteristic feature of isotope shifts in many nuclei [20]. A solution to this problem has eluded since long. It can be seen from the figure that the RMF theory is successful in reproducing this kink. We would like to stress that the RMF theory provides a first-ever microscopic description of anomalous isotopic shifts in Sr nuclei. Such an anomalous behaviour is a generic feature of deformed nuclei which include almost all isotopic chains in the rareearth region [20. It is to be remarked that here also the RMF theory is shown to have a remarkable success [8]. This is amply demonstrated through Fig.6 where the isotope-shift of rare-earth nuclei with atomic numbers ranging from $Z=60$ upto $Z=70$ are shown.

In short the RMF theory is highly successful in describing the ground state properties 
( e.g binding energies, nuclear radii, the deformations, isotope shifts etc). The agreement with the experiment is really remarkable. It is to be stressed that the RMF theory uses limited number of parameters as compared to other theories and models and at the same times it provides a consistent and unified description of the ground state properties of nuclei over range of isospin.

\section{$3 \quad$ Program structure and test run}

The code consists of the fortran program and two additional files: DIZ.PAR and DIZ.DAT. The file DIZ.PAR contains the relevant information regarding the dimensions, depending upon the number of oscillator shells to be included. The standard form of DIZ.PAR is for 12 shells both for fermions and bosons $\left(N_{F}=N_{B}=12\right)$. The input file DIZ.DAT provides the necessary information relevant to the specific case being calculated (the details are attached). The program runs interactively. After three iterations the user has to provide as input the number of iterations and the value of the parameter xmix. As an example for hundred iterations and the value of $\mathrm{xmix}=0.3$ the user should write -100 and in the next 0.3. The main program DIZ calls various subroutines reading the data and performing the execution. The operation consists essentially of two parts. The first part using DIZ.DAT starts the program, initializes and generates all the relevant information. It uses the subroutines PREP, READER, START, DEFAULT, GAUSS. The fortran file DIZLIB contains several general subroutines required at various stages in the program. The second part is the main part and does the entire execution using the initial information provided by the first part. The iterative procedure is carried out by the subroutine ITER. In the first iteration it solves the Dirac equation using the potential terms calculated by the initial guessed values of the fields. The solutions (Dirac spinors) are used to calculate the sources which in turn are used in solving the Klein Gordon equations. These solutions are used in the next iteration for the solution of the Dirac equation. This procedure continues till the convergence of the desired accuracy is obtained. In this operation ITER calls various subroutines like POTGH, DIRAC, FIELD, OCCUP, DENSIT, EXPECT etc. The output file (DIZ.OUT) is prepared in RESU and INOUT. The user is advised to rerun the program once more after getting the convergence. This will make the output file DIZ.OUT compact. The file DIZ.OUT contains explicit headings to make it selfexplainatory. Several detailed comments are introduced at various places in the program which helps the user to understand the different functions of subroutines and at the same time to figure out what is going on at important steps of the program.

For an illustration DIZ.DAT file is listed for the specific case of ${ }^{88} \mathrm{Sr}$. A part of the output file DIZ.OUT is also listed providing the results of the RMF calculation for this nucleus, using the parameter set NL-SH. If ones wishes to use a different set of Lagrangian parameters then the new set of parameters can be inserted in place of the corresponding numbers of the NL-SH set in the subroutine DEFAULT. 


\section{Acknowledgement}

Support from the Bundesministerium für Forschung und Technologie under the project 06 TM734 (6) is acknowledged. One of the authors (G.A.L) acknowledges support by the European Union under the contract TMR-EU/ERB FMBCICT-950216.

\section{References}

[1] B.D. Serot and J.D. Walecka, Adv. Nucl. Phys. 16 (1986) 1.

[2] B.D. Serot, Rep. Prog. Phys. 55 (1992) 1855.

[3] Y.K. Gambhir, P. Ring and A. Thimet, Ann. Phys. (N.Y.) 194 (1990) 132.

[4] M.M. Sharma, G.A. Lalazissis, and P. Ring, Phys. Lett. B317 (1993) 9.

[5] M.M. Sharma, M.A. Nagarajan, P. Ring, Ann. Phys. (N.Y.) 231 (1994) 110.

[6] M.M. Sharma, G.A. Lalazissis, W. Hillebrandt, and P. Ring; Phys. Rev. Lett. 72 (1994) 1431

[7] G.A. Lalazissis and M.M. Sharma, Nucl. Phys. A586 (1995) 201.

[8] G.A. Lalazissis, M.M. Sharma and P. Ring, Nucl. Phys. A597 (1996) 35.

[9] W. Pannert, P. Ring and J. Boguta, Phys. Rev. Lett. 59 (1987) 2420.

[10] Y.K. Gambhir and P. Ring, Phys. Lett. 202B (1988) 5.

[11] J. Boguta and A.R. Bodmer, Nucl. Phys. A292 (1977) 413.

[12] P. Möller, J.R. Nix, W.D. Myers, and W.J. Swiatecki, Atomic Data and Nuclear Data Tables 59 (1995) 185.

[13] M. Abramowitz and I. A. Stegun, "Handbook of Mathematical Functions", Dover, New York, 1970.

[14] Y.K. Gambhir and P. Ring, Mod. Phys. Lett. 8 (1993) 787.

[15] D. Vautherin, Phys. Rev. C 7 (1973) 296.

[16] J. Decharge and D. Gogny, Phys. Rev. C 21 (1980) 1568.

[17] M.M. Sharma, M.A. Nagarajan, and P. Ring, Phys. Lett. B 312 (1993) 377.

[18] G. Audi and A.H. Wapstra, Nucl. Phys. A565 (1993) 1.

[19] Y. Aboussir, J.M. Pearson, A.K. Dutta, and F. Tondeur, Atomic Data and Nuclear Data Tables 61 (1995) 127. 
[20] E.W. Otten, in Nuclear Radii and Moments of Unstable Nuclei, in Treaties on Heavy-Ion Science, (ed. D.A. Bromley) Vol. 7 (Plenum, N.Y. 1988) p. 515.

[21] F. Buchinger, E.B. Ramsay, E. Arnold, W. Neu, R. Neugart, K. Wendt, R.E. Silverans, P. Lievens, L. Vermeeren, D. Berdichevsky, R. Fleming, D.W.L. Sprung and G. Ulm, Phys. Rev. C41 (1990) 2883.

[22] S. Raman, C.H. Malarkey, W.T. Milner, C.W. Nestor, and P.H. Stelson, At. Data Nucl. Data Tables 36 (1987) 1.

[23] G.D. Alkhazov, A.E. Barzakh, V.P. Denisov, V.S. Ivanov, I.Ya. Chubukov, N.B. Buyanov, V.S. Letokhov, V.I. Mishin, S.K. Sekatskii, and V.N. Fedoseev; JETP Lett. 48 (1988) 413. 


\section{Figure Captions.}

Fig. 1 The calculated RMF binding energy per nucleon for Sr isotopes obtained with the parameter set NL-SH. The predictions from the mass models FRDM and ETF-SI are also shown for comparison.

Fig. 2 The quadrupole deformation $\beta_{2}$ obtained from relativistic Hartree minimization for $\mathrm{Sr}$ isotopes using the force NL-SH. The predictions of the mass models FRDM and ETF-SI are also displayed for comparison. Nuclei exhibiting a shape coexistence and thus a second minimum in the RMF theory are depicted by a square surrounding the $\beta_{2}$ value of the lowest minimum.

Fig. 3 The prolate-oblate shape coexistence for neutron-rich $\mathrm{Sr}$ isotopes predicted in the RMF theory. The energy difference in the prolate and oblate minima for Sr isotopes is shown.

Fig. 4 The calculated rms charge and neutron radii of $\mathrm{Sr}$ isotopic chain obtained by using the force NL-SH.

Fig. 5 The calculated and the experimental [20, 21] isotope shifts for Sr isotopes.

Fig. 6 The calculated and the experimental isotope shifts for Nd, Sm, Gd, Dy, Er, Yb nuclei. The empirical values for all but $\mathrm{Gd}$ nuclei are taken from ref. [20]. The isotope shifts for Gd nuclei have been derived from ref. [23]. 
Table 1: The parameters of the force NL-SH. All the masses are in $\mathrm{MeV}$, while $g_{2}$ is in $\mathrm{fm}^{-1}$. The other coupling constants are dimensionless.

\begin{tabular}{ccccc}
\hline \hline $\mathrm{M}=939.0$ & $m_{\sigma}=526.059$ & $m_{\omega}=783.0$ & $m_{\rho}=763.0$ & \\
& & & & \\
$g_{\sigma}=10.444$ & $g_{\omega}=12.945$ & $g_{\rho}=4.383$ & $g_{2}=-6.9099$ & $g_{3}=-15.8337$ \\
\hline \hline
\end{tabular}


Table 2: The quadrupole deformations $\beta_{2}$ for $\mathrm{Sr}$ isotopes obtained in the RMF theory using the force NL-SH. The FRDM and ETF-SI predictions are also listed. The available empirical deformations (expt.) obtained from the $\mathrm{BE}(2)$ values are also given in the last column. The experimental values do not depict the signature of the deformation. The deformations of nuclei showing a shape coexistence with a second minimum are given in the parentheses.

\begin{tabular}{|c|c|c|c|c|c|}
\hline $\mathrm{A}$ & NL-SH & $\overline{\text { FRDM }}$ & ETF-SI & expt. & \\
\hline 72 & 0.324 & 0.371 & $\begin{array}{c}-0.30 \\
\end{array}$ & - & \\
\hline 74 & 0.430 & 0.400 & 0.44 & - & \\
\hline 76 & 0.450 & & 0.421 & 0.44 & - \\
\hline 78 & 0.450 & 0.421 & 0.43 & 0.434 & \\
\hline 80 & 0.402 & 0.053 & 0.40 & 0.377 & \\
\hline 82 & -0.200 & 0.053 & -0.30 & 0.290 & \\
\hline 84 & 0.089 & 0.053 & 0.15 & 0.211 & \\
\hline 86 & 0.0 & 0.053 & 0.00 & 0.128 & \\
\hline 88 & 0.0 & 0.045 & 0.00 & 0.117 & \\
\hline 90 & -0.058 & 0.045 & -0.11 & - & \\
\hline 92 & $0.181(-0.165)$ & 0.080 & -0.15 & - & \\
\hline 94 & $0.230(-0.218)$ & 0.255 & -0.19 & - & \\
\hline 96 & $0.356(-0.275)$ & 0.338 & 0.35 & - & \\
\hline 98 & $0.424(-0.309)$ & 0.357 & 0.39 & 0.354 & \\
\hline 100 & $0.426(-0.314)$ & 0.368 & 0.38 & 0.372 & \\
\hline 102 & $0.413(-0.295)$ & 0.369 & 0.40 & - & \\
\hline 104 & $0.403(-0.277)$ & 0.361 & 0.38 & - & \\
\hline
\end{tabular}




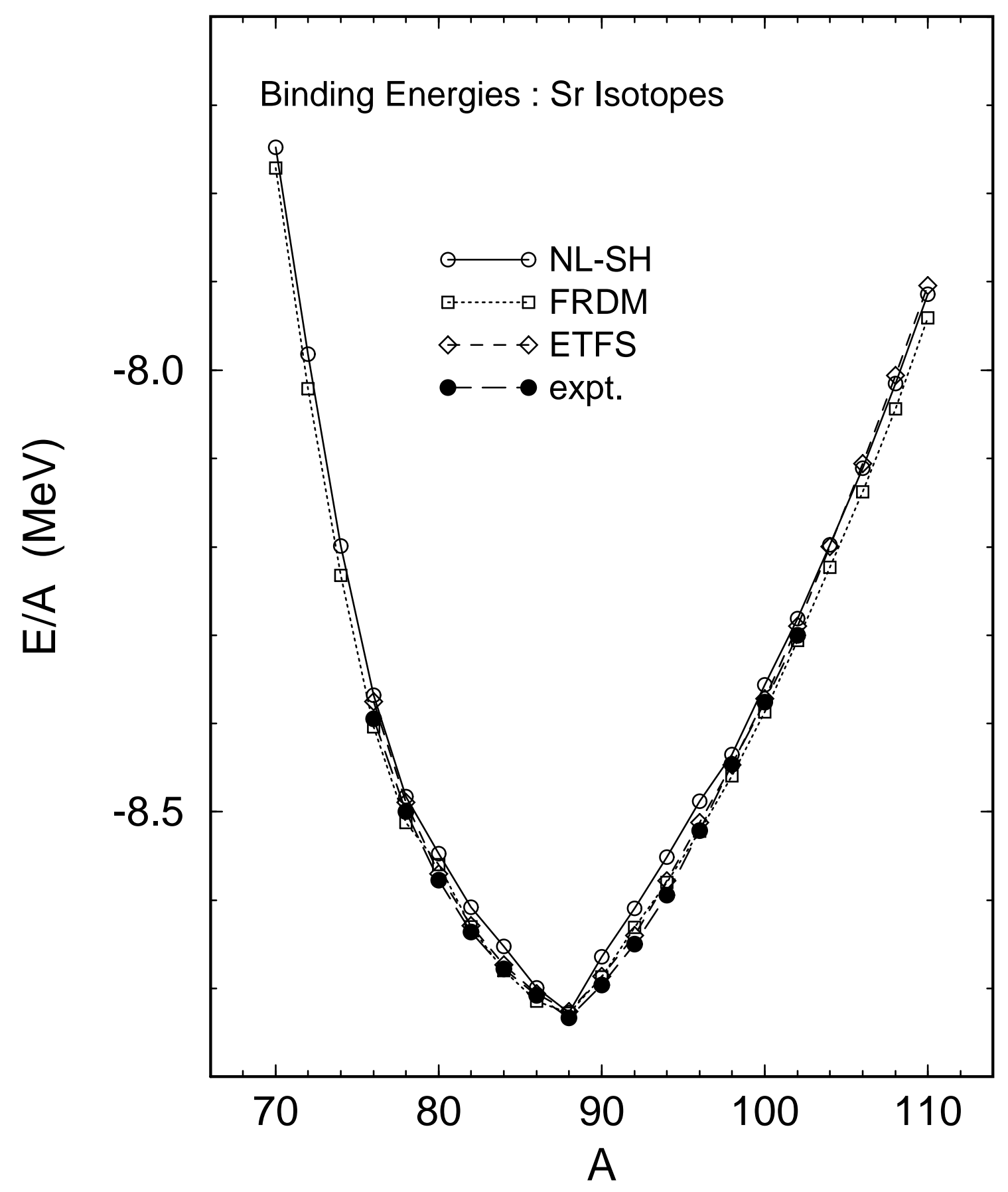

Figure 1 


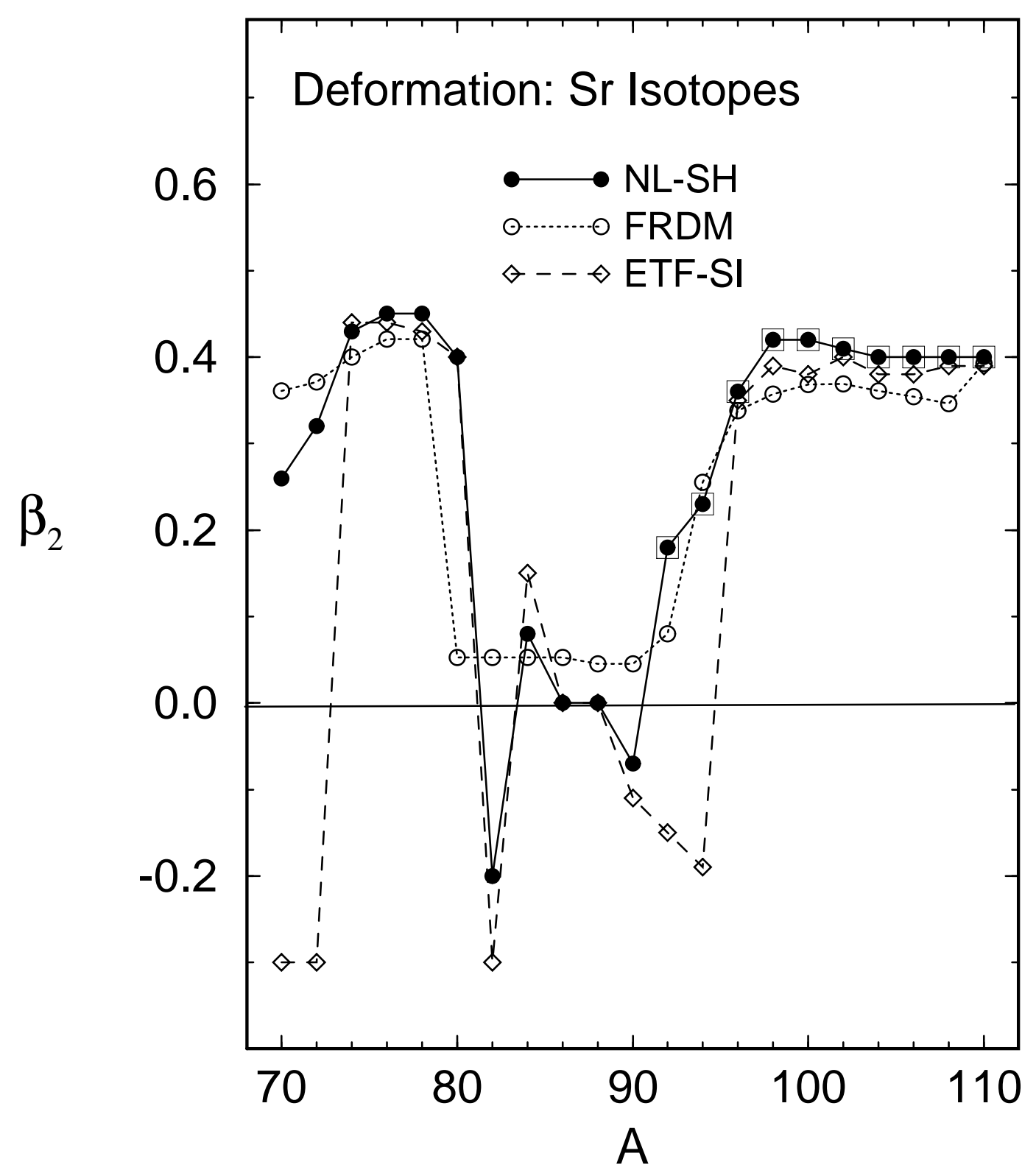

Figure 2 


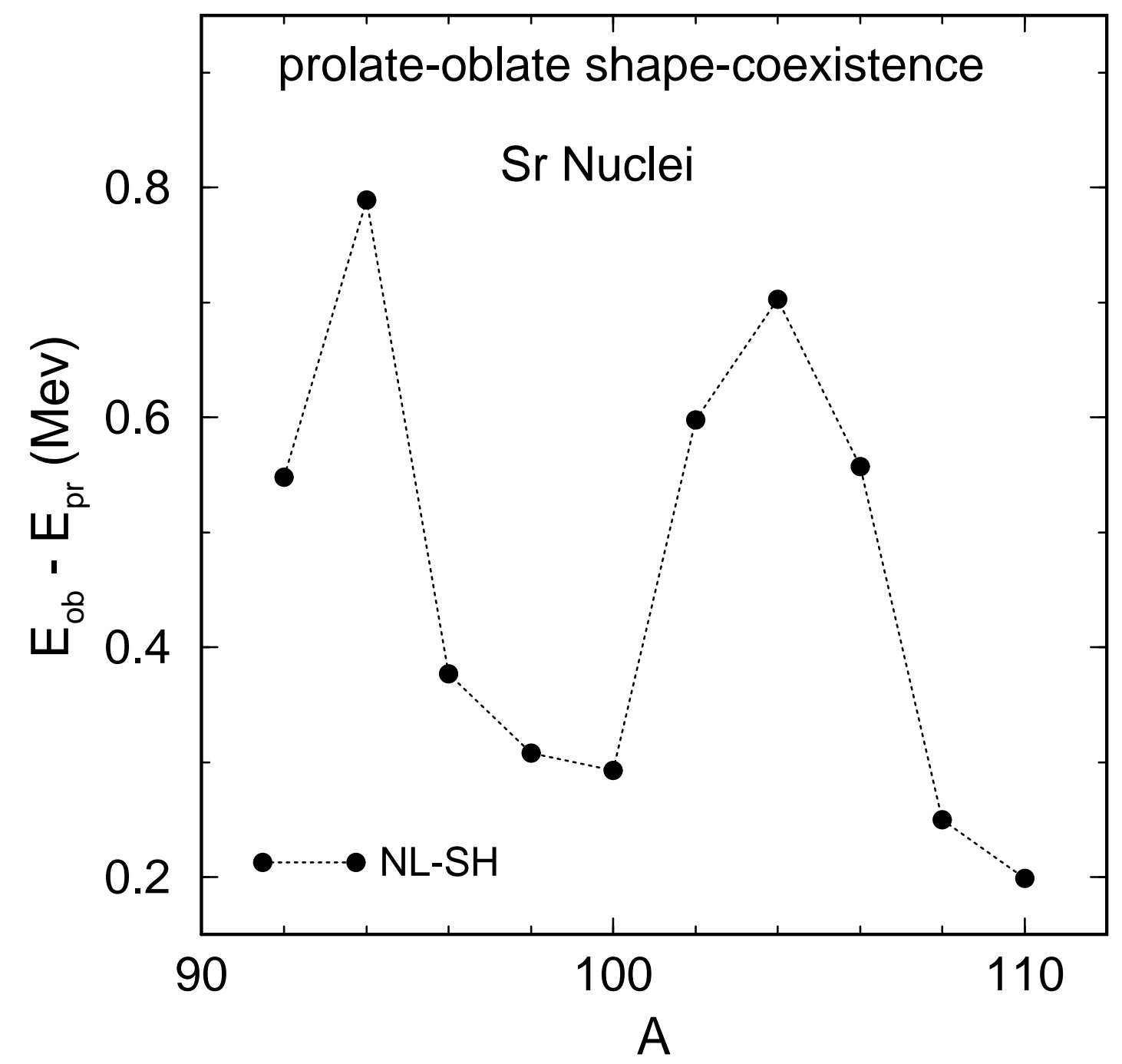

Figure 3 


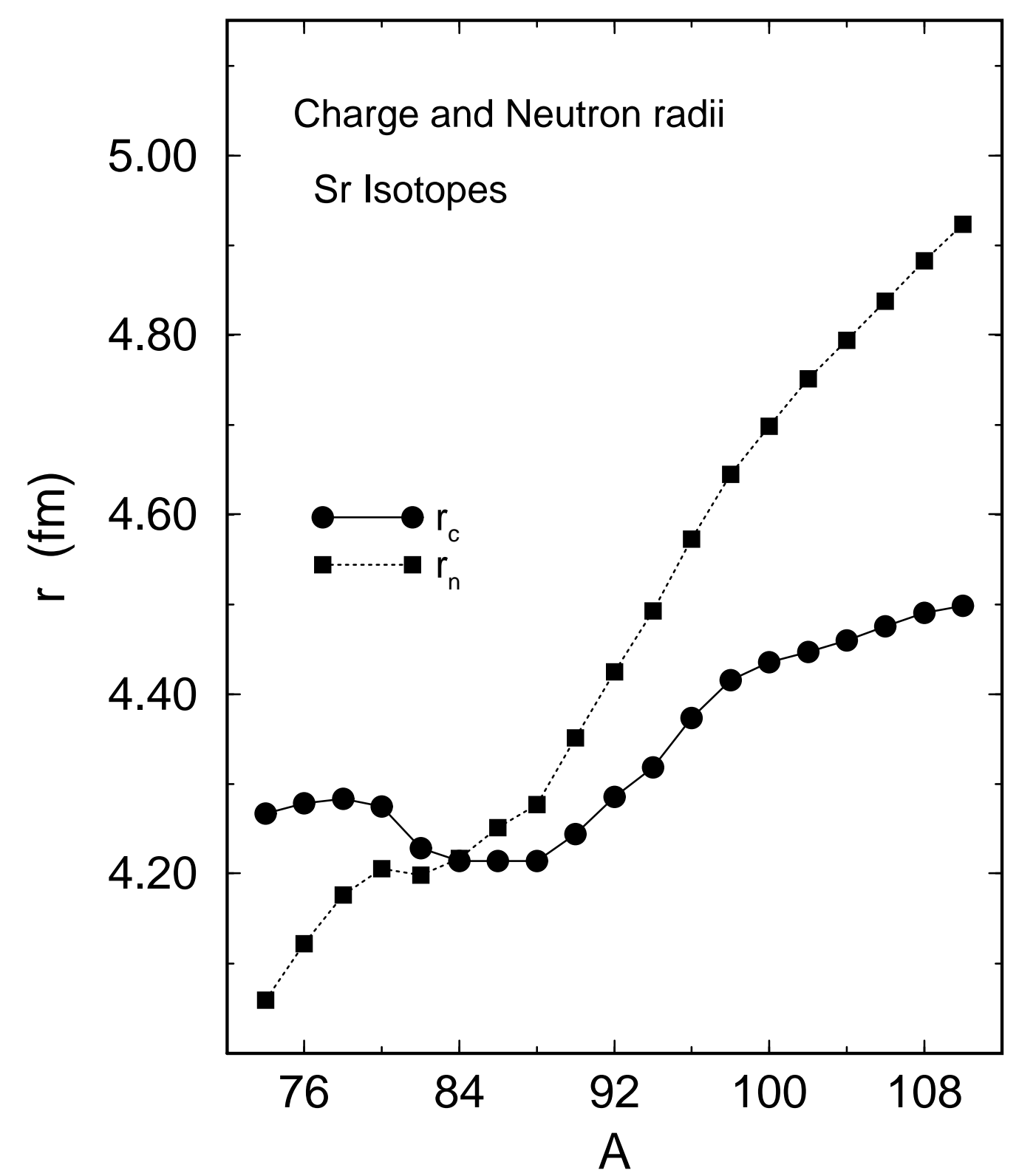

Figure 4 


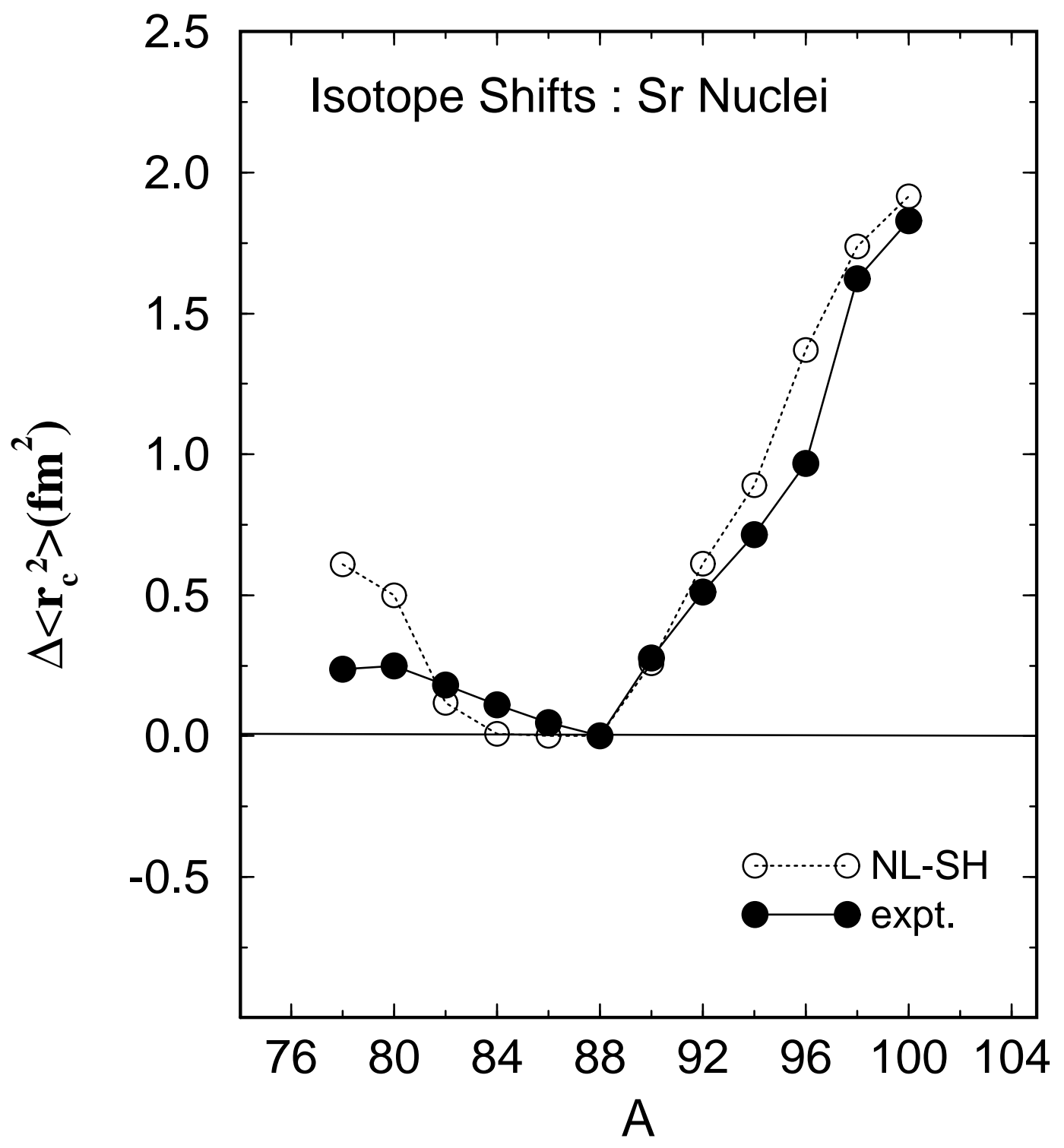

Figure 5 

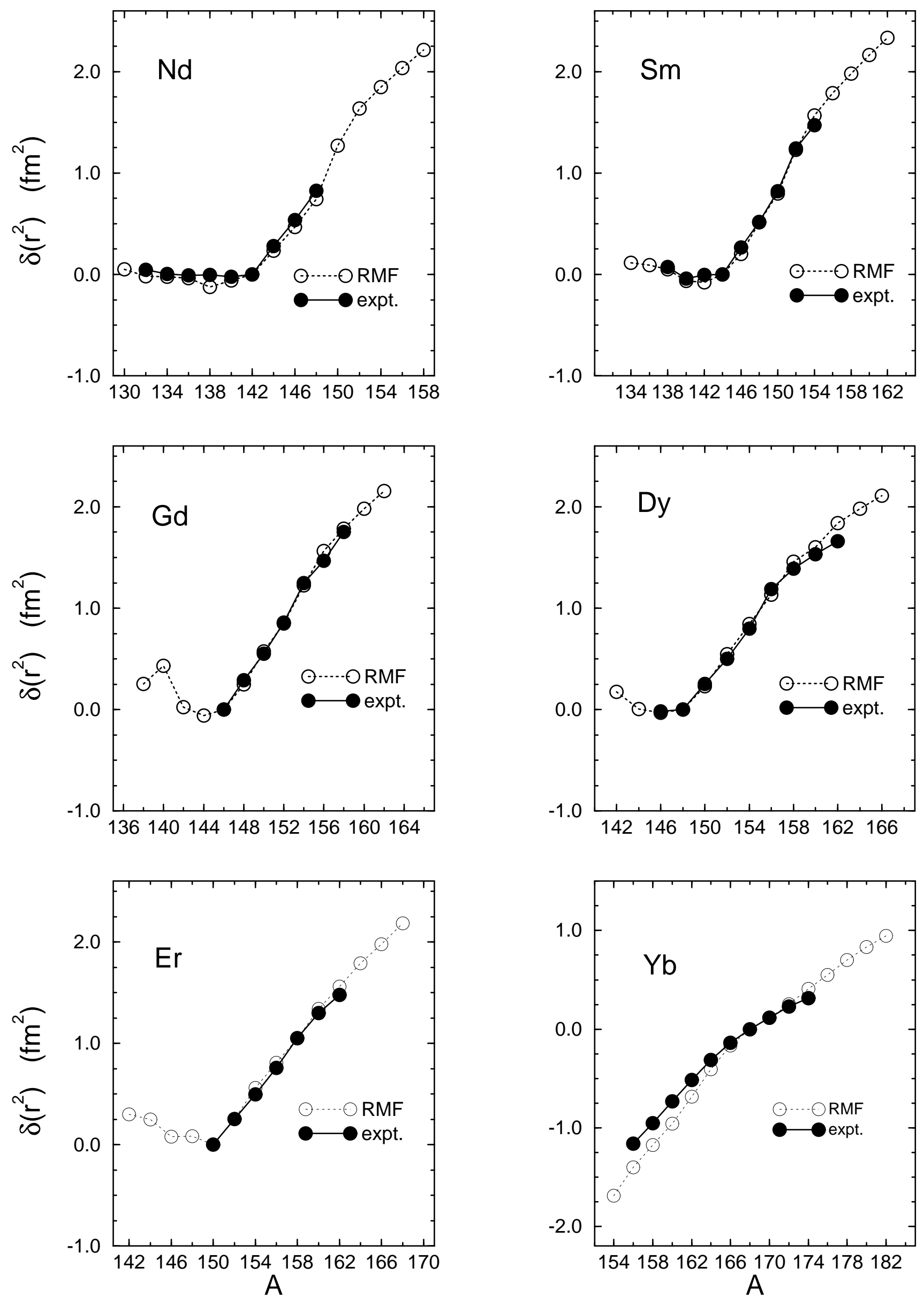

Figure 6 